\title{
Histoire et phénomènes religieux chez les Asna de l'Ader (pays hausa, République du Niger)
}

History and religious phenomena among the Asna of Ader (Hausa, Niger)

Nicole Échard

\section{(2) OpenEdition \\ Journals}

Édition électronique

URL : http://journals.openedition.org/span/156

DOI : $10.4000 /$ span. 156

ISSN : 2268-1558

Éditeur

École pratique des hautes études. Sciences humaines

\section{Édition imprimée}

Date de publication : 1 septembre 1975

Pagination : 63-77

ISSN : 0294-7080

\section{Référence électronique}

Nicole Échard, « Histoire et phénomènes religieux chez les Asna de l'Ader (pays hausa, République du Niger) ", Systèmes de pensée en Afrique noire [En ligne], 1 | 1975, mis en ligne le 03 juillet 2013, consulté le 19 avril 2019. URL : http://journals.openedition.org/span/156 ; DOI : 10.4000/span.156 


\section{HISTOIRE ET PHENOMENES RELIGIEUX CHEZ LES ASNA DE L'ADER}

(Pays hausa. République du Niger)

par Nicole ECHARD

PROPOS : Notes sur les transformations de la pensée religieuse et l'utilisation du système religieux dans l'interprétation des faits historiques. Ne sont reprises ici que quelques données qui ont spécifiquement trait aux phénomènes religieux et qui sont incluses dans les traditions historiques des paysans hausa de l'Ader (1). L'approche procède d'une mise en corrélation entre données portant sur le système religieux et données portant, d'une part sur l'organisation sociale, d'autre part sur le passé des groupes ayant constitué la société paysanne hausa de l'Ader.

Le terme asna a actuellement, en pays hausa, une connotation essentiellement religieuse et désigne les non musulmans. $Y$. Urvoy (2) supposait que ce terme désignait une ethnie particulière dont l'origine géographique aurait été l'Ader. Dans cette région, en effet, et bien que le terme ait dans certains contextes une acception religieuse, il semble que se soit constituée, à date ancienne, une société que $1^{\text {'on }}$ peut dire Asna et qui aurait été contemporaine, dans sa genèse, de certains Etats hausa, en particulier le Gobir. La société Asna résulta de la juxtaposition dans l'espace de communautés villageoises autarciques. Elle ne semble pas admettre de continuité entre l'organisation sociale qu'elle s'est donnée et toute forme de centralisation politique. Ce hiatus s'est manifesté tout au

(1) Traitées dans L'expérience du passé, histoire de la société paysanne hausa de l'Ader (Rêpublique du Niger), Etudes Nigériennes, à paraître.

(2) Histoire des poputations du Soudan Central (Colonie du Niger) Paris, Larose, 1936. 
Iong de son histoire et son organisation semble avoir étè suffisante pour participer à l'échec des tentatives centralisatrices de certains groupes de migrants. Du point de vue des idéologies religieuses, aucun mythe n'ayant jamais organisé de manière contraignante le système religieux, celui-ci s'est constitué à partir de la pratique des nombreux groupes en présence, laquelle sous-tend une pensẻe en perpétuelie mouvance et à caractère de plus en plus totalisant.

Au travers des traditions historiques relevées en Ader, détenues par les groupes ayant constitué les communautés villageoises, apparaissent certaines des transformations de la pensée religieuse dont les éléments sont utilisés, par ailleurs, pour rendre compte de faits historiques. Actuellement, des bribes de la vieille religion Asna, pour une part "religion de la nature", sont encore présents mais un culte de possession, dit bori, a pris une large extension et s'oppose à I'Islam qui ne s'est développé réellement qu'au xxème siècle. Culte de possession boxi et Islam renvoient à des projets de société non seulement différents mais à bien des égards antinomiques.

1). Le temps de I'histoire, sans faire l'objet d'une réelle périodisation, n'en est pas moins perçu comme constitué de trois espaces temporels qui se sont succédés bien que variant a'un groupe à l'autre et d'une micro-région à 1 'autre (l) et qui sont :

- Un temps mythique, ahistorique, correspondant à l'émergence et à l'existence souterraine des groupes dits asnan ramu, "Asna des trous", "Asna des Cavernes". Semblables à des dieux, en ayant certaines caractêristiques et parlant leur langue, les Asna des Cavernes étaient différenciés des plantes et des animaux. Certains de ceux-ci, habitant des terriers, vivaient en symbiose avec eux et sont parfois censés

(1) La représentation du temps (ainsi, d'ailleurs, que l'utilisation du système religieux dans l'interprétation historique) fait l'objet d'un bref développement dans un article à parâtre in Cahiers d'Etudes Africaines, écrit en collaboration avec P. Bonte et portant sur la conception du passé des Twareg Kel Gress et des Hausa de 1'Ader. 
les avoir engendrés. Ils se nourrissaient de cueiliette ainsi que du pillage des termitières et des fourmilières. Lorsque les réserves alimentaires etaient épuisées, les dieux partaient à ia recherche de vivres.

L'univers était perşu come constitué de deux parties: la leur, connue, souterraine, lieu d'un certain nombre d'espẻces animales, de dieuy ; 1 'autre. étrangère, extérieure, aomaine de créatures inconnues (hommes et animaux ne fouissant pas le sol) et de plantes.

- A ce temps mythigue succéda l'époque historique des premières fondations. Les traditions la concernant font état du passage d'une économie de cueillette à une économie agricole, impliquant une société fixée dans des villages dont le terroir environnant est mis en valeur. Lä constitution de ces premières communautés villageoises - dont l'ensembie allait former la société Asna - se fait selon un schéma dualiste dont nots ne retiendrons ici que les éléments qui font apparaître le rôle du système religieux.

Se différenciant dafinitivement d'avec les dieux et accédant ainsi à l'humanité, les Asna des Cavernes s'ailient à ảes Chasseurs spşcialisés qui se dêplaçaient à la recherche de gibier et pratiquaient sans doute l'agriculture itinerante. I'alliance est scellée par un échange : en contre-partie au savoir technique des chasseurs - habitat, tissage, agriculture - , les Asna des Cavernes donnent des dieux et des objats de culte, c'est-ä-äire ur savoir religieux. Les groupes s'installent dans un rapport de complémentarité, se répartissant les fonctions et fondant les premières communautés, constituées chacune de deux quartiers distincts. La reproduction de l'alliance repose sur l'exercice des fonctions déterminées par les origines et. s'appuie sur la pratique religieuse de chacun des groupes. Ies cultes mis en place ont en effet, entre autres rôles, d'assurer le rappel périodique des origines et de la mise en place de l'alliance, toute transformation du rituel sauvée de l'oubli renvoyant à l'évolution du rapport entre ies deux groupes constituants initiaux. 
- Le troisième espace temporel est celui des fondations postérieures. De nombreux migrants, venus principalement de l'AIr, du Gobir et du Kurfey - région proche da Songhay - s'installent en Ader. A l'exception des migrants "chefs" qui tentèrent de constituer des chefferies, ils reproduisent la mème organisation dualiste lors de la fondation de leurs communautés villageoises. Le stock de dieux dont ils disposent est constitué de divinités, soit liées à leur groupe et ayant voyagé avec lui, soit trouvées sur le site où ils s'installent.

Cette troisième époque s'accompagne de l'insertion de groupuscules de migrants dans les communautés fondées antérieurement à leur arrivée en Ader. L'évolution de la société se fait essentiellement par les ajustements successifs des groupes constituants les uns aux autres, la dynamique sociale se fondant sur les relations entre groupes dominants-fondateurs des communautés villageoises. Les principales transformations semblent avoir été liêes à celle - progressive - des chasseurs jadis itinérants inclus dans ces communautés d'agriculteurs sédentaires. Elles sont actuellement en partie interprétées et exprimées par le recours au système religieux. Il est impossible de déterminer si ce mode d'interprétation des faits historiques est récent ou non, si les traditions historiques ont changé de contenu ou non. Tout au plus peuton constater, d'une part que le compte-rendu actuel fait du passé tend à utiliser de manière de plus en plus importante des éléments du système religieux, d'autre part que l'expression de faits historiques récents - liés à la colonisation et à l'indépendance nationale - passe de manière privilégiée par le canal de la religion.

2). Le système religieux, tel qu'il peut être observé actuellement, semble s'être élaborê à partir de la multiplicité des usages religieux des groupes constituants : différences entre les rituels même si ceux-ci sont, dans certains cas, très sommaires; grande diversité de dieux, parfois à large extension, parfois liés à un seul groupe ou à un lieu gêographique. Ces traits engendrent divers types de relations avec les dieux. 
L'expression religieuse, à l'origine formée des cultes dits Asna, 's'est, jusqu'à nos jours, enrichie et diversifiée par la mise en place (courant XVIIIème siècle ?) de ce qu'il est convenu d'appeler "culte de possession", bori, et par le développement de I'Islam (Xxème siècle).

Au temps reputé comme etant celui des origines, lors de la mise en place des rituels rappelant l'alliance entre les Asna des Cavernes et les Chasseurs, il semble qu'ait prévalu une conception dualiste de l'univers qui aboutissait à la répartition sulvante'

- Monde tellurique souterrain incluant les eaux "du dessous" mais aussi les eaux "du dessus, d'en haut", du ciel.

Lieu des Asna des Cavernes.

D'ou : ceux-ci seront responsables des rapports entre les hommes et, $d$ 'une part la climatologie (chutes et répartition des pluies, déroulement des saisons), d'autre part la production de la vie (germination, fécondité humaine ....)

- Monde superficiel, compris entre le monde souterrain et les eaux superieures.

Lieu des Chasseurs.

D'où : ceux-ci régissent principalement les rapports entre les hommes et les plantes/les animaux/les autres hommes/ainsi que certaines divinités uniquement liées au culte de possession.

On peut faire de cette représentation le grossier schéma ci dessous :

$$
\text { eaux d'en haut }
$$

vide

superficie

monde souterrain souffles, vents

hommes, animaux, plantes 
C'est dans un espace vide, intercalaire entre la surface de la terre - à laquelle sont rivés hommes, animaux et plantes - et les eaux superieures que se meuvent les souf-. fles : iska, "le vent", terme dont le pluriel, iskoki, désigne les dieux. Cette représentation, encore présente actuellement, est utilisée dans certaines pratiques magico-religieuses.

Il ressort de cette bipartition que les Chasseurs sont associés à l'organisation des rapports parentaux et sociaux, c'est-à-dire voués à un rôle de première importance. Par ailleurs, et conformément à ce schéma, les traditions attestent que, dès les origines, les chasseurs détenaient, outre un savoir technique, la connaissance des plantes et, ce faisant, de la thérapeutique. Les souvenirs conservés à propos de la transformation des groupes de chasseurs font état d'une spécialisation de certains d'entre eux qui, après avoir renoncé à certaines chasses, se sont constitués en groupe de guerriers et de thérapeutes. C' est à l'intérieur de ces derniers que se serait développé le culte de possession bori qui est, actuellement, le lieu par excellence du savoir médicinal. Cette relation entre culte de possession et Chasseurs est manifeste au travers d'un certain nombre de données autres, par exemple :

- L'immense majorité des adeptes et les détenteurs des fonctions associées au culte de possession sont issus de lignages de chasseurs. Ce n'est que ces cinq dernières années que des membres d'autres groupes ont eu accès aux fonctions de prêtrise, ce fait étant lié à l'évolution sociale contemporaine où l'opposition Chasseurs/Asna des Cavernes n'est plus déterminante.

- Les mentions concernant la religion dans les traditions d'origine, nombreuses pour les cultes des Asna des Cavernes, sont presque inexistantes en ce qui concerne le culte de possession. Quand elles existent, elles donnent des indications sur une origine importée du culte et le situent du côté des chasseurs, ou des "fils des femmes" par rapport aux premiers occupants définis comme "fils des hommes", position occupée par les Asna des Cavernes.

- Les tensions qui se sont produites, à partir surtout du 
XIXème siècle, entre chasseurs et Asna des Cavernes sont exprimés par des rivalités religieuses, les Asna des Cavernes tendant à s'approprier ou à promouvoir des rituels impliquant la séquence transe-possession et, inversement, des adeptes du culte de possession tendant à reprendre en charge des cultes à des aivinités de la nature spêcifiques aux Asna des Cavernes.

Le développement de l'Islam, présent depuis une époque très ancienne (fin XVIIème siècle) mais qui ne connut une réelle expansion qu'à partir de la seconde partie du XIXème siècle, clarifie pour une part les données du système religieux par le biais de la réduction des identifications qu'il a opérée. La seule confrêrie bien représentée au début du xxème siècle était la Tijanya marquée, en ce qui concerne ce propos, par une grande tolérance à l'égard des manifestations religieuses non musulmanes (1). La confrérie qadriah se développa par la suite et participa à la diffusion d'un Islam plus rigoriste, répressif à l'égard des non musulmans et ... des femmes. Le Nyassisme, pourvu d'un projet social, bien implanté dans une vallée de l'Ader, est condamné par de nombreux musulmans et a fait l'objet d'une répression gouvernementale dans les premières années qui ont suivi l'indépendance nationale. Actuellement, on constate une opposition musulmans/non musulmans qui recouvre et exprime des divergences idéologiques, sociales et économiques. L'Islam a participé à l'introduction de la notion de propriété privée, de pouvoir économique fondé sur le commerce et la thésaurisation, bref à l'introduction d'un certain nombre de notions fondant l'inégalité entre des groupes d'hommes (et entre les sexes), véhiculant un projet de société très différent de celui propre aux paysans de l'Ader. L'affrontement se fait sur le plan religieux, les musulmans s'opposant de plus en plus activement aux adeptes des cultes Asna et de possession.

3). Ci-dessous deux exemples illustrant le rapport système religieux-interprétation historique au travers des traditions d'origine de certains groupes.

(1) cf. l'exposé de Aghroum Abd Erraouf. 
a) Manipulation des dieux : exemple des Follakawa et des Katamawa (pays Follakam).

--- Les chasseurs Follakawa ("les gens du Follakam") qui savaient construire des paillotes, s'allient aux

- Asna des Cavernes Katamawa qui avaient quatre yeux deux devant et deux derrière la tête - et quatre divinités

- Les Katamawa gardent deux divinités, D1 et D2, "qui travaillent pour les cultures",

- D1 - Divinité féminine de lieu liée à la germination et à l'eau.

- D2 - Divinité féminine de la classe des dogwa, ensemble de divinités sorcières de brousse considérées comme autochtones qui, de plus, "font le bori" en Ader.

Les dogwa sont attestêes dans le culte de possession de l'ensemble du pays hausa et en Afrique du Nord (A.J.N. Tremearne) ainsi que dans le culte songhay des holey (J. Rouch).

- D1 et D2 : culte conjoint annuel avant la saison des pluies.

et donnent les deux autres, D3 et D4, aux Follakawa

- D3 - Divinité féminine liée aux Follakawa dont la cruauté la fait parfois considérer comme une dogwa.

- D4 - Divinité féminine attestée dans l'ensemble du pays hausa et, parfois, dans le Songhay.

- Les Follakawa demandent aux Katamawa de renoncer aux deux yeux de derrière la tête. Les Katamawa "laissent" alors les deux yeux de derrière aux divinités D1 et D2.

--- Courant XVIIème, les Follakawa luttent contre une chefferie voisine fondée par des migrants. D3 et D4 "travaillent pour la guerre". D3 "ne laisse pas vivant un seul jeune homme en pleine force".

--- D2 épouse D5, génie du culte de possession bori, attesté dans une grande partie du pays hausa sous divers noms.

--- Au XIXème siècle, des migrants Gobiwara cultivateurs, chassés du Gobir par les guerres peules (Jihad d'Usman dan Fodyo), arrivent dans le Follakam et font alliance avec les Follakawa.

- Les Follakawa donnent aux Gobirawa D3 "parce que c'est un génie guerrier comme eux". 
- Les Gobirawa donrent aux Follakawa D6: qu'ils ont amenë avec eux et qui leur est lié.

- Les heurts entre Follakawa et Gobirawa s'expriment par les menaces faites par les Gobirawa de restituer D3, "la méchante". Les Follakawa disent : "Nous sommes trop contents de nous en être débarassés, gardez-la pour vous!"

Au travers de cet exemple apparaissent les traits suivants : - La dẹtermination des relations inter-groupes est exprimée par des transferts de divinités.

- Chacun des deux groupes initiaux est pourvu d'une divinité féminine limitée soit à un lieu (D1), soit au groupe (D3) et d'une divinité ayant une très large extension. (D2 et D4).

- Les divinités données aux Follakawa changent d'affectation et deviennent des divinités "travaillant pour la guerre". - L'apparition du culte de possession entraine le mariage de la divinité de lieu avec un génie du culte de possession.

b) Transformation des rituels : exemple des Tarimawa et des Gazurawa (village de Bagey)

--- Les chasseurs Gazurawa s'allient aux Asna des Cavernes Tarimawa pour fonder le viliage de Bagey.

- La caverne des Tarimawa était constituée d'une caverne dite "maison" d'où partaient quatre galeries souterraines dont deux se prolongeaient jusqu'à aboutir à des lieux précịs. Les Asna des Cavernes vivaient souterrainement avec leurs deux divinités, Tarima - d'oú leur nom de Tarimawa, "ceux de Tarima" - et son époux Bakin Aljani, "Génie Noir", et une hyène tachetée. Après leur sortie restent dans la caverne les divinités et l'hyène tachetée.

-- Les Asna des Cavernes fondent pour une part leur alliance avec les Gazurawa sur l'accomplissement de rituels destinés à assurer le bon déroulement du cycle agraire.

- Le premier rituel a lieu pendant la saison dite bazara chaude et progressivement de plus en plus humide, qui précède la saison des pluies.

- Le second rituel, considéré comme le plus important, a lieu après la germination du mil pendant la première partie de la saison des pluies.

Ces rituels ont le même déroulement et comportent deux parties:

- Déambulation souterraine, avec les instruments néces- 
saires au culte, a partir de la caverne "maison" jusqu'au lieu de culte ou débouche l'une des galeries.

- Offrandes sur le lieu de culte :

- Bouillie de mil préparée sur place, répandue sur le lieu, le reste étant réparti entre les enfants et 1 'hyène tachetée.

- Un pagne blanc pour Tarima.

- Un boubou en tissu indigoté pour Bakin Aljani.

- Un animal pour chacune des divinités dont une part de la viande est réservée à l'hyène tachetée.

Par la suite, les Chasseurs mettent en place un rituel dit "ouverture de la brousse", pratiqué dans l'ensemble du pays hausa, exécuté en saison sèche et froide, qui comporte deux phases :

- Déambulation selon un itinéraire fixe à la recherche de gibier : ouverture de la période de chasse.

- Mise à mort d'une hyène rayée à l'issue de ce périple. Les entrailles de l'hyène rayée sont examinées à des fins divinatoires.

--- Courant XVIIIème siècle : l'hyène tachetée de la caverne disparait. Elle est remplacée par l'hyène rayée chassée lors de la cérémonie d'ouverture de la brousse. L'hyène tachetée est parfois dite être devenue le génie du culte de possession Kure. Le rituel des Asna des Cavernes se modifie en conséquence.

--- Bakin Aljani est actuellement considéré, par certains Tarimawa et Gazurawa, comme Arne, divinité du culte de possession. Tarima, qui a une devise jouée par les musiciens du bori, entralne parfois des possessions.

Cet exemple fait apparaitre :

- Les fondements de l'alliance au travers des rituels mis en place, laquelle situe les groupes dans un rapport de complémentarité et d'opposition (sec/humide, souterrain/superficiel, rappel des apports des chasseurs : pagne et boubou, divination du côtê des chasseurs etc...)

- La transformation des relations : l'animal chassé par les chasseurs s'installe dans la caverne, c'est à dire s'accapare une part du monde souterrain. La relation des deux groupes évolue vers une importance de plus en plus grande des Chasseurs.

- Enfin, la récupération des divinités des Asna des Cavernes par le culte de possession qui s'est développé parmi les chasseurs. Ce faisant, le culte à Tarima et Bakin Aljani disparait. 


\section{DISCUSSION}

J. GUTWIRTE Où se trouve l'articulation entre l'expression religieuse et ies faits historiques? Comment la détermine-t-on ?

N. ECHARD Les traditions historiques conservees par la sociétë paysanne constituent une sorte de corpus de récits formalisés. Ceux-ci rendent compte, - au travers de divers types d'interprétation fondés souvent sur l'usage du système religieux -, des principaux moments de la genèse de la société et des principaux évẽnements qui ont eu une incidence sur sa transformation. A partir de la seconde moitié du xIXème siècle environ, les faits historiques ne font plus l'objet d'un tel traitement. Il n'en reste au mieux qu'un compte rendu anecdotique qui $n$ 'apprend rien au niveau de l'histoire globale, qui n'est inséré dans aucun schème interprétatif. L'interprétation et le souvenir du fait historique passent à partir de ce moment par les canaux de l'expression religieuse. Dans le culte de possession, en particulier, certains dieux sont des signifiants sociohistoriques. On peut se demander s'il y a incapacité de la société à formaliser, conceptualiser, bref à comprendre; des faits s'inscrivant dans un contexte à la fois plus large et different de ce qu'il était auparavant. On peut se demander si ces faits ne requièrent pas un nouveau type de réflexion, avant qu'une formulation acceptable soit possible. Il semble bien que la pensée religieuse, de nature totalisante, ait, pour une part, pris en charge cette reflexion et c'est dans cette mesure que le culte de possession apparaft comme un lieu privilégié de l'analyse des faits socio-historiques. Le culte de possession, comme 1'Islam, permet une très large participation des membres de la société à l'expression religieuse. A l'inverse de l'Islam, le culte de possession tend à traverser la société en ce qu'il ne prend pas en compte, pour l'intégration des adeptes, les différences de sexes, d'âge ou de statut social. 
J. GUTWIRTH Quelle est la situation du christianisme dans l'Ader ?

N. ECHARD La première mission catholique implantée dans I'Ader date de 1965. Ses.missionnaires ainsi que ceux de la mission protestante américaine ne font que de la présence évangélique.

J. ROUCH Nous venons de faire une enquête sur les saisons en pays zarma. Toutes les traditions disent qu'une grande fête religieuse où on demande la pluie doit avoir lieu. le loème jour du 7 ème mois, c'est-à-dire le loème jour 7 mois après la dernière pluie. Ceci impose un certain calendrier pour que recommence le cycle. Or, il semblerait que le calendrier suivi se fonde sur l'observation de certaines plantes. Il semblexait aussi que les saisons soient déterminées par le rôle que jouent certaines familles de divinités. Tu as montré comment, dans le temps et dans l'espace, s'est operée une certaine stratification. $Y$ aurait-il une stratification liée aux saisons?

N. ECHARD J'ignore s'il y a une relation entre la manifestation de certains génies et les saisons. Il ne le semble pas. Toutefois, certaines divinités zarma, récemment apparues, sont liées à des saisons. Ainsi, les génies qui ont pris la place des sorciers et manipulent la foudre. Il est hors de question de les appeler pendant la saison des pluies.

J. ROUCH Il semble que la plupart des génies de possession Songhay soient des personnages historiques, héros ou individus morts de mort violente, - tués par des sorciers ou dont la mort ne correspondait pas à la norme -, dont l'âme errante venait hanter le souvenir des survivants et qui, donc, pouvaient se manifester dans le cadre déjả institutionnalisé de la danse de possession. Une de mes hypothèses de travail est le rôle positif du sorcier qui consommerait la force vitale de la personne et donnerait le double à ses "collègues", génies de la possession, remettant ainsi la victime en circulation. Actuellement, au Niger, on assiste à l'arrivée de nouvelles divinités qui sont ces génies "froids", ces "fantômes". 
I1 serait intéressant de voir comment une divinité stéréotypée Songhay se transforme en passant dans l'Ader, pourquoi un même personnage mythique joue un rơle différent selon les lieux, enfin pour quelles raisons certains personnages ont une telle puissance d'exportation.

M. CARTRY Y a-t-il d'autres cultes de possession lies a d'autres entités que les bori?

N. ECHARD Le terme bori, dêsigne non le génie mais tout à la fois 1 'état de possession, la divinité, l'institution même du culte. Il y. a un énorme brassage de tous les dieux disponibles qui passent d'une classe dans l'autre, se mettant tout à coup a "faire le bori".

L. DE HEUSCH Peut-on conclure que la possession est apparue à une époque relativement récente?

N. ECHARD Les auteurs donnent à cette question des réponses variables. Ainsi, A.J.N. Tremearne (1) écrit que le culte de possession hausa est pré-islamique. J.Broustra (2), qui a mené une recherche dans la vallée de Maradi au Niger, le présente comme s'étant développé dans une situation de crise, face à l'Islam militant de la fin du XIXème siècle. En Nigéria, le culte de possession est interdit depuis trop longtemps pour qu'on puisse en juger. Dans la Vallée de Maradi, il est marginal - ce qui n'est pas le cas en Ader - ne concernant que les femmes et ne comportant que des cérémonies intéressant l'individu. De plus, le panthéon sur lequel il s'appuie dans la Vallée de Maradi semble assez restreint. J. Broustra indique au'une partie des dieux seraient d'anciens dieux claniques qui, auparavant, déterminaient déjà chez leurs prêtres des états de transe et de possession.

En ce qui concerne l'Ader, le culte de possession semble lié à. la transformation des groupes de chasseurs et la séquence transe et possession serait attestée depuis le XVIIIème siècle. Rien ne permet de déterminer quand, à partir de cette séquence, s'est constitué le culte institutionnalisé 
connu actuellement.

M. CARTRY Le fait que des dieux claniques se transforment en génies de possession est une hypothèse intéressante. On en aperçoit des traces en pays Gourmantché : des puissances, liées à la notion de fétiche, qui étaient propitiées par des prêtres de lignage, tendent actuellement à devenir des génies possédant les fidèles. On pourrait ainsi "saisir sur le vif" le passage d'un état à un autre. C'est là un phénomène très récent en pays Gourmantché où le culte de possession est peu répandu.

L. DE HEUSCH Les anciens dieux des Asna des Cavernes ont-ils conservé une certaine autonomie ou bien sont-ils tous passës dans le bori?

N. ECHARD Certains dieux sont encore spécifiquement liés à ce qu'on a appelé "la nature". Toutes les situations sont possibles : il $y$ a des dieux qui, après avoir "fait le bori" un certain temps, ont disparu du culte; il y a certains dieux qui sont actuellement en train de commencer à "faire le bori", d'autres qui ont une devise jouée par les musiciens du culte mais ne possédent personne, d'autres enfin qui n'ont aucun attribut hormis leur nom et qui possédent des adeptes.

J. ROUCH Tous les cultes dont tu as traité sont-ils à superposer ?

N. ECHARD Il ne s'agit pas d'une "superposition" mais plutôt d'une sorte de "combinatoire" qui implique, pour son élucidation, la prise en compte des principaux traits de l'organisation sociale.

J. ROUCH Cela ne voudrait-il pas dire que I'Ader a manqué d'un "génie de synthèse" du type de celui des songhay qui jamais n'ont. fait disparaitre un culte, une ethnie, qui ont admis la diversité et l'ont intégrée dans le mythe et qui pensent que c'est cet ensemble de diversités qui constituait l'Etat Songhay ?

N. ECHARD Ce qui fait le dynamisme de la pensée religieuse hausa en Ader, ce qui fait l'intérêt de ces signifiants que sont les divinités, c'est précisément l'aptitude du système idéologicoreligieux à se transformer au fur et à mesure de l'évolution socio-historique. 
En d'autres termes, s'il y avait eu, en raison de la prèsence de ce "génie de synthèse", par exemple production d'un mythe exerçant une contrainte certaine sur l'expression religieuse, il est peu probable que la pensée religieuse en Ader eût acquis les caractéristiques soulignées ici. 